\title{
MicroRNA-505-3p inhibits development of glioma by targeting HMGB1 and regulating AKT expression
}

\author{
ZHENLIN $\mathrm{CHENG}^{1}$, BIN WANG ${ }^{1}$ and CHENG ZHANG $^{2}$ \\ ${ }^{1}$ Department of Neurosurgery, Zhangye People's Hospital Affiliated to Hexi University, Zhangye, Gansu 734000; \\ ${ }^{2}$ Department of Neurosurgery, People's Hospital of Xinjiang Uygur Autonomous Region, Urumqi, Xinjiang 830001, P.R. China
}

Received March 20, 2019; Accepted November 14, 2019

DOI: 10.3892/ol.2020.11714

\begin{abstract}
Previous studies have reported that microRNA (miR)-505 exhibits important effect in human cancers. However, the regulatory mechanism of miR-505-3p/high-mobility group box 1 (HMGB1) axis is still unclear in glioma. Therefore, the regulatory mechanism of miR-505-3p/HMGB1 axis in glioma was illuminated. Expression of miR-505-3p and HMGB1 was observed by RT-qPCR. Protein expression was measured by western blot analysis. Dual luciferase assay was performed to confirm the relationship between miR-505-3p and HMGB1. The function of miR-505-3p was investigated by MTT and Transwell assays. Expression of miR-505-3p was reduced in glioma, which was related to poor clinical outcomes and prognosis in glioma patients. Moreover, overexpression of miR-505-3p suppressed proliferation, migration and invasion of glioma cells. In addition, HMGB1 was confirmed as a direct target of miR-505-3p, and miR-505-3p inhibited the development of glioma by targeting HMGB1. Furthermore, miR-505-3p blocked EMT suppressing p-AKT expression in glioma cells. In conclusion, miR-505-3p inhibited the development of glioma by targeting HMGB1 and regulating AKT expression.
\end{abstract}

\section{Introduction}

Gliomas are malignant tumors derived from neuroepithelial tissue and are the most common malignant tumors of the central nervous system, accounting for 40-50\% of intracranial tumors (1). According to the WHO classification, gliomas are divided into I-IV grades. Among them, astrocytoma and polar glioblastoma are common, followed by oligodendymal glioma and medulloblastoma (2). Malignant glioma generally refers to gliomas of grades III and IV, with a high recurrence rate and poor prognosis (3). Moreover, the recurrence of glioma

Correspondence to: Dr Cheng Zhang, Department of Neurosurgery, People's Hospital of Xinjiang Uygur Autonomous Region, 91 Tianchi Road, Tianshan, Urumqi, Xinjiang 830001, P.R. China

E-mail: zonp9t48690865@163.com

Key words: miR-505-3p, HMGB1, glioma, AKT is also a major threat to the health of patients. The recurrence time of glioma depends on the degree of differentiation and treatment. Glioma patients receiving conventional treatments usually relapse within 1 to 5 years (4). Because the tumor has not been completely resected or regenerated, a large proportion of gliomas will recur after surgery. Therefore, it is necessary to actively search for effective diagnosis and treatment.

In recent years, microRNAs (miRNAs/miRs) have been reported to regulate multiple biological activities of human cancers by blocking the expression of their target genes (5). In particular, the function of many miRNAs has been identified in glioma. For example, miR-124-3p inhibited cell proliferation and motility by targeting EphA2 in gliomas (6). In addition, miR-499a was found to inhibit cell proliferation and promote apoptosis in glioma through inactivating Notch1 and the MAPK signaling pathway (7). In contrast, miR-423-3p was demonstrated to promote tumor growth of glioma by targeting PANX2 (8). Recently, the specific role of miR-505-3p in human diseases caught our attention. For example, miR-505 was identified as a potential biomarker for the imatinib response in patients with chronic myeloid leukemia (9). It has been reported that miR-505 identified endothelial cell migration and tube formation in patients with essential hypertension (10). Moreover, miR-505 was found to inhibit growth factor-induced epithelial mesenchymal transition (EMT) (11). However, the regulatory mechanism of miR-505-3p remains unclear in glioma, and needs to be further elucidated.

As a member of the HMGB superfamily, high-mobility group box 1 (HMGB1) has been found to regulate DNA organization and gene transcription (12). Furthermore, HMGB1-mediated expression of matrix metalloproteinase-9 was found to promote tumor cell invasiveness in non-small cell lung cancer (13). In addition, it was found that HMGB1 silencing inhibited cell proliferation and invasion of MGC-803 gastric cancer cells (14). Overexpression of HMGB1 was correlated with tumor progression and poor prognosis in human colorectal carcinoma (15). Knockdown of HMGB1 has been found to inhibit cell proliferation and to induce apoptosis in hemangioma by blocking AKT pathway (16). It was also reported that miR-1231 exerted tumor suppressive effects by regulating the EGFR/PI3K/AKT axis in glioma (17). However, the molecular mechanism of miR-505-3p/HMGB1/AKT axis remains unclear in glioma. 
In this study, the alteration of miR-505-3p expression was first identified in glioma tissues and cell lines. In addition, the relationship between miR-505-3p and HMGB1 was confirmed. The regulatory mechanism of miR-505-3p/HMGB1/AKT was also investigated in glioma cells. These results will help improve the diagnosis and treatment of gliomas.

\section{Patients and methods}

Glioma specimens. In total, 44 human glioma tissues were acquired from the Department of Neurosurgery, Zhangye People's Hospital Affiliated to Hexi University (Zhangye, China). Glioma tissue samples were classified according to the World Health Organization (WHO) standards. Gliomas (11 of the 44) were classified as low-grade (5 WHO I and 6 WHO II, diffuse astrocytoma) and 33 were classified as high-grade (19 WHO III and 14 WHO IV, anaplastic astrocytoma). Clinicopathological information of the patients with glioma included in the present study is summarized in Table I. Eight samples of normal brain tissues were obtained from internal decompression patients undergoing surgical operation. The patients with glioma did not receive any treatment prior to surgery. The tissues were frozen in liquid nitrogen and then stored at $-80^{\circ} \mathrm{C}$ in a refrigerator. Informed consents were obtained from the patients. The study was approved by the Institutional Ethics Committee of Department of Neurosurgery, Zhangye People's Hospital Affiliated to Hexi University.

Cell culture. The U251 (BNCC337874), A172 (BNCC341782), LN229 (BNCC341218) glioma cell lines and normal human astrocyte NHA cells (BNCC341796) were acquired from Cell Bank of the Chinese Academy of Sciences. Glioma cells were cultured in Dulbecco's modified Eagle's medium (DMEM; Thermo Fisher Scientific, Inc.) supplemented with $10 \%$ fetal bovine serum (FBS) (Thermo Fisher Scientific, Inc.) at $37^{\circ} \mathrm{C}$ in a humidified incubator containing $5 \% \mathrm{CO}_{2}$. NHAs were cultured using AGM Astrocyte Growth Medium Bullet kit (Lonza) containing astrocyte growth media and supplements.

Cell transfection. The miR-505-3p mimics (5'-GGGAGC CAGGAAGUAUUGAUGU-3') or inhibitor (5'-ACUACUGAG UGACAGUAGA-3') and negative control (NC, 5'-UUCUCC GAACGUGUCACGUTT-3') were obtained from GenePharma. Then they were transferred into U251 cells, respectively, with Lipofectamine 2000 (Invitrogen; Thermo Fisher Scientific, Inc.) based on the protocols of the manufacturers. After $48 \mathrm{~h}$ of transfection, the effcacy was determined by RT-qPCR analysis.

RT- $q P C R$ analysis. Total RNA was extracted from the tissues and cells using TRIzol reagent (Invitrogen; Thermo Fisher Scientific, Inc.). Complementary DNA was synthesized by RevertAid RT Reverse Transcription kit (Thermo Fisher Scientific, Inc.). PCR amplifcation was performed by SYBR ${ }^{\circledR}$ Green qPCR Assay kit (Thermo Fisher Scientific, Inc.) on ABI 7500 Fast system (Applied Biosystems; Thermo Fisher Scientific, Inc.) with primers. U6 or GAPDH was used as control for miR-505-3p or HMGB1. Relative miRNA or mRNA expression was calculated using the $2^{-\Delta \Delta \mathrm{Cq}}$ method, normalized against U6 or GAPDH and then compared with
Table I. Relationship between miR-505-3p expression and their clinicopathological characteristics of glioma patients.

\begin{tabular}{lcrrr}
\hline & & \multicolumn{2}{c}{ miR-505-3p } & \\
\cline { 3 - 3 } Characteristics & Cases & High & Low & P-value \\
\hline Age (years) & & & & 0.205 \\
$\geq 55$ & 24 & 10 & 14 & \\
$<55$ & 20 & 7 & 13 & \\
Sex & & & & 0.361 \\
Male & 26 & 11 & 15 & \\
Female & 18 & 6 & 12 & \\
Tumor size (mm) & & & & 0.284 \\
$\leq 5.0$ & 16 & 6 & 10 & \\
$>5.0$ & 28 & 11 & 17 & \\
Necrosis & & & & $0.032^{\mathrm{a}}$ \\
Yes & 14 & 5 & 9 & \\
No & 30 & 12 & 18 & \\
WHO grade & & & & $0.019^{\mathrm{a}}$ \\
I-II & 11 & 6 & 5 & \\
III-IV & 33 & 11 & 22 & \\
\hline
\end{tabular}

Statistical analyses were performed by $\chi^{2}$ test. ${ }^{\mathrm{a}} \mathrm{P}<0.05$ was considered significant. WHO, World Health Organization.

the control group (18). The primer sequences used in qPCR were as follows: miR-505-3p forward, 5'-CTACGTGGGTCACCC CCTC-3' and reverse, 5'-CCAAAGGAGACCTCGTAGT-3'; and U6 forward, 5'-GCTTCGGCAGCACATATACTAAA-3' and reverse, 5'-GCTTCACGAATTTGCGTGTCAT-3'. HMGB1 forward, 5'-TATGGCAAAAGCGGACAAGG-3' and reverse, 5'-CTTCGCAACATCACCAATGGA-3'; GAPDH forward, 5'-ACAACTTTGGTATCGTGGAAGG-3' and reverse, 5'-GCCATCACGCCACAGTTTC-3'.

Western blot analysis. The protein samples were obtained using RIPA lysis buffer. The protein concentration was detected using a BCA protein kit (Beyotime). A total of $50 \mu \mathrm{g}$ of protein was separated by $10 \%$ SDS-PAGE and transferred to a polyvinylidene fluoride (PVDF) membrane (Bio-Rad Laboratories, Inc.). The membrane was then blocked using 5\% skim milk and incubated with E-cadherin (Rabbit monoclonal; dilution, 1:1,000; cat. no. ab1416; Abcam), N-cadherin (Rabbit polyclonal; dilution, 1:1,000; cat. no. ab18203; Abcam), Vimentin (Rabbit polyclonal; dilution, 1:1,000; cat. no. ab137321; Abcam), MMP-2 (Rabbit polyclonal, dilution, 1:300; cat. no. 10373-2-AP; Proteintech), MMP-9 (Rabbit polyclonal, dilution, 1:600; cat. no. 10375-2-AP; Proteintech), AKT (Rabbit polyclonal; dilution, 1:1,000; cat. no. ab8805; Abcam), p-AKT (phospho S473, Rabbit monoclonal; dilution, 1:1,000; cat. no. ab81283; Abcam), HMGB1 (Rabbit monoclonal; dilution, 1:1,000; cat. no. ab227168; Abcam) and GAPDH (Rabbit monoclonal; dilution, 1:1,000; cat. no. ab181602; Abcam) antibodies overnight at $4^{\circ} \mathrm{C}$. After washing, the membrane was washed and incubated with horseradish peroxidase-conjugated secondary antibodies (dilution, 1:5,000; cat no. ab190492; 

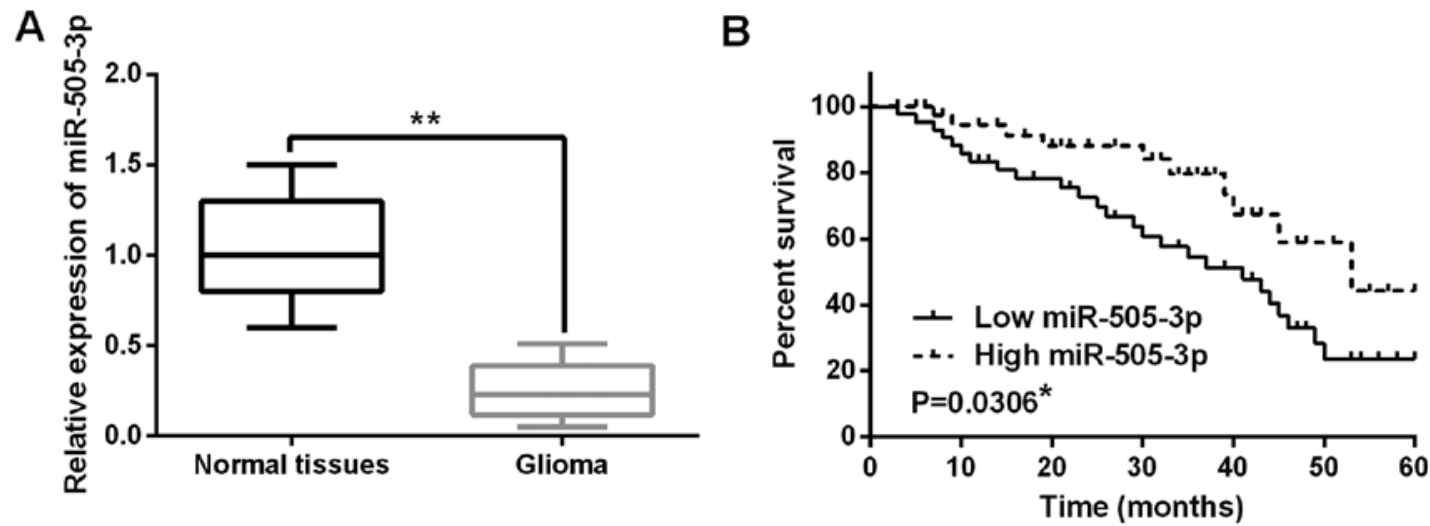

Figure 1. Expression of miR-505-3p was reduced in glioma. (A) Expression of miR-505-3p in glioma tissues. (B) High miR-505-3p expression was correlated with longer overall survival in glioma patients. ${ }^{*} \mathrm{P}<0.05,{ }^{* *} \mathrm{P}<0.01$.

Abcam) for $2 \mathrm{~h}$ at $37^{\circ} \mathrm{C}$. The protein bands were visualized using ECL (Pierce; Thermo Fisher Scientific, Inc.).

MTT assay. To analyze cellular proliferation, $5 \times 10^{3} \mathrm{U} 251$ cells with miR-505-3p mimics or inhibitor were cultured in a 96-well plate with $100 \mu \mathrm{l}$ of DMEM containing $0.5 \mathrm{~g} / 1$ MTT (Thermo Fisher Scientific, Inc.). Then, U251 cells were cultured at $37^{\circ} \mathrm{C}$ for $12,24,48$ or $72 \mathrm{~h}$, after which the medium was removed. Next, $50 \mu 1$ dimethyl sulfoxide (Thermo Fisher Scientific, Inc.) was added. Following incubation at $37^{\circ} \mathrm{C}$ for $10 \mathrm{~min}$, the absorbance at $490 \mathrm{~nm}$ of each sample was determined using a plate reader (Bio-Rad Laboratories, Inc.). All experiments were run in triplicate.

Transwell assay. Transwell chambers $(8-\mu \mathrm{m}$ pore size membranes) were employed to perform cell migration and invasion assays. For detection of cell migration, $5 \times 10^{3}$ cells transfected with miR-505-3p mimics or miR-505-3p inhibitor were resuspended in $500 \mu 1$ of medium without FBS and placed in the upper chambers. The lower chamber was filled with medium containing $10 \%$ FBS as a chemoattractant. For cell invasion, the upper chambers were coated with Matrigel (BD Biosciences). After $24 \mathrm{~h}$ at $37^{\circ} \mathrm{C}$, the non-migrating or non-invading cells on the top well were gently removed. The cells on the lower surface of the membrane were fixed with $70 \%$ ethanol and stained with $0.1 \%$ violet (Sigma-Aldrich; Merck KGaA). Finally, the number of removed cells were counted using a microscope (Olympus Corporation). All experiments were run in triplicate.

Bioinformatics prediction. TargetScan version 7.1 online software (www.targetscan.org) was used to predict the potential targets of miR-505-3p, according to the manufacturer's instructions. Briefly, 'human' was selected as the target species, and miR-505 was inserted as the investigated miRNA. miR-505-3p was predicted to be able to directly bind to the seeding sequences of the 3'-UTR of HMGB1.

Dual luciferase assay. The 3'-UTR of wild-type or mutant HMGB1 was inserted into pcDNA3.1 plasmid vector (Promega Corporation) to perform luciferase reporter experiments. Then, the luciferase vector and miR-505-3p mimics were transfected into U251 cells using Lipofectamine 2000 and incubated for
$48 \mathrm{~h}$. Finally, the luciferase activity was measured through dual luciferase assay system (Promega Corporation). All experiments were run in triplicate. The U251 cells with NC and luciferase vector were used as the control.

Statistical analysis. Data are shown as mean \pm SD and were analyzed using SPSS 19.0 (SPSS, Inc.) or Graphpad Prism 6 (GraphPad Software, Inc.). The association between miR-505-3p and clinicopathological features in glioma patients was calculated by Chi-square test. Differences between multiple groups were calculated by one-way ANOVA followed by Tukey's post hoc test. Pearson's correlation analysis was performed to examine the correlation between the miR-505-3p and HMGB1 expression in glioma tissues. Survival curves were plotted by Kaplan-Meier analysis, and log-rank test was used to compare survival differences. $\mathrm{P}<0.05$ was considered to indicate a statistically significant difference.

\section{Results}

Expression of miR-505-3p is reduced in glioma. The expression of miR-505-3p was detected in glioma tissues by RT-qPCR assay. The expression of miR-505-3p was reduced in the 44 cases of glioma tissues compared to normal tissues (Fig. 1A). The median relative expression level of miR-505-3p in glioma tissues was taken as a cut-off point to separate low and high expression of miR-505-3p. Moreover, miR-505-3p was also found to be associated with WHO grade $(\mathrm{P}=0.019)$ and necrosis $(\mathrm{P}=0.032$; Table I). In addition, we found that low miR-505-3p expression was associated with shorter overall survival in glioma patients $(\mathrm{P}=0.0306$; Fig. 1B). These findings suggest that miR-505-3p may be involved in the pathogenesis of glioma.

miR-505-3p restrains proliferation, migration and invasion of glioma cells. Expression levels of miR-505-3p were also measured in U251, A172, LN229 and NHA cell lines. Downregulation of miR-505-3p was also detected in U251, A172 and LN229 cell lines compared to NHA cells (Fig. 2A). miR-505-3p mimics or inhibitor was then transfected into U251 cells to investigate its function in glioma cells. RT-qPCR assay showed that miR-505-3p expression was increased by miR-505-3p mimics and decreased by miR-505-3p inhibitor 
A
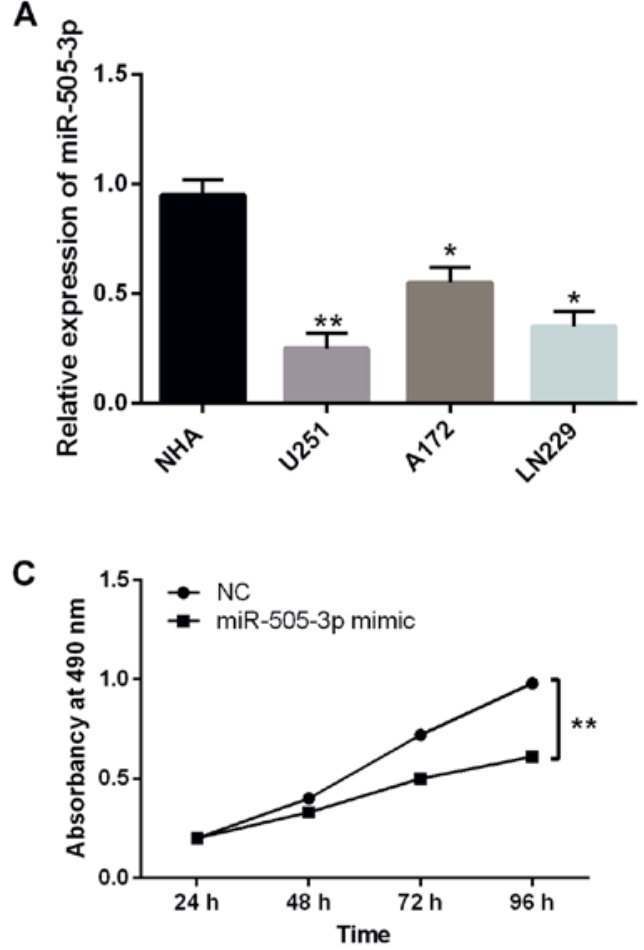

E

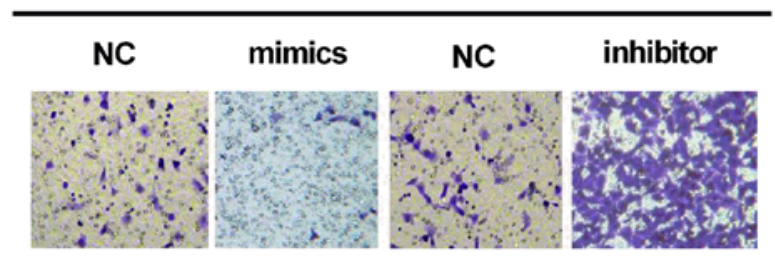

$\mathbf{F}$

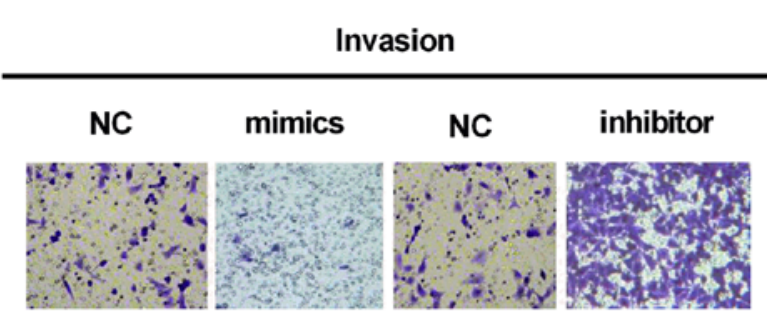

B

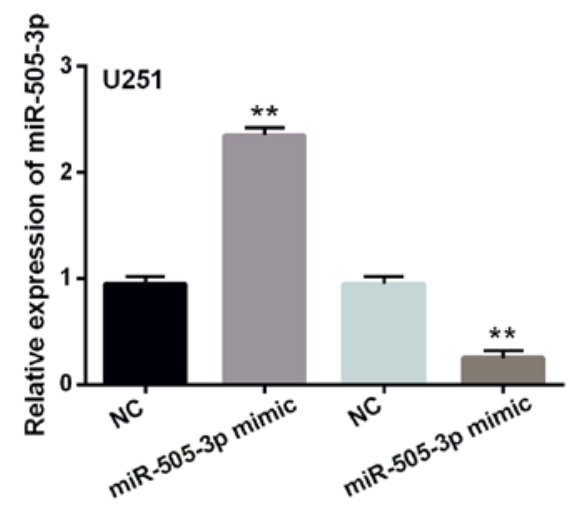

D
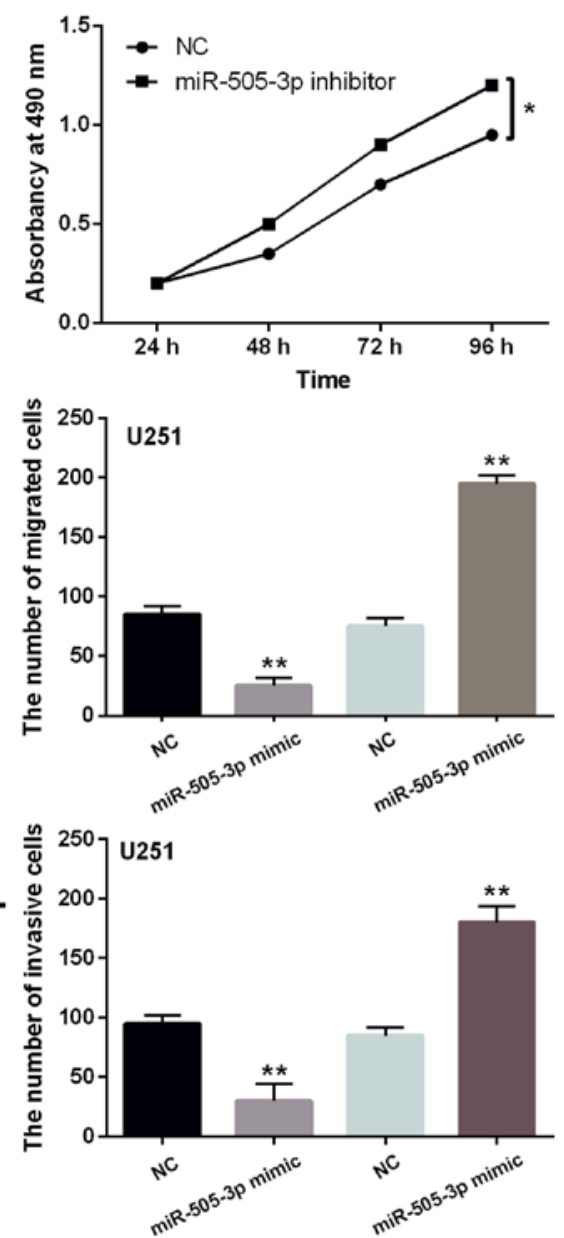

Figure 2. miR-505-3p restrains proliferation, migration and invasion of glioma cells. (A) miR-505-3p expression in U251, A172, LN229 and NHA cell lines. (B) Expression of miR-505-3p was examined in U251 cells with miR-505-3p mimics or inhibitor. (C and D) Cell proliferation was measured in cells containing miR-505-3p mimics or inhibitor. (E and F) Cell migration and invasion analysis in cells containing miR-505-3p mimics or inhibitor. ${ }^{*} \mathrm{P}<0.05$, ${ }^{* * *} \mathrm{P}<0.01$.

(Fig. 2B). Functionally, upregulation of miR-505-3p inhibited proliferation of U251 cells (Fig. 2C). In contrast, downregulation of miR-505-3p was found to promote cell proliferation in U251 cells (Fig. 2D). As with the above results, overexpression of miR-505-3p inhibited cell migration in U251 cells, whereas downregulation of miR-505-3p promoted migration of U251 cells (Fig. 2E). Moreover, similar effect of miR-505-3p on cell invasion was also identified in U251 cells (Fig. 2F). Based on these results, miR-505-3p might have inhibitory effect on the development of glioma.
HMGB1 is a direct target of miR-505-3p in glioma. In addition, TargetScan database (http://www.targetscan.org/) shows that HMGB1 has a binding site with miR-505-3p (Fig. 3A). Next, luciferase reporter assay was performed to confirm the prediction. We found that miR-505-3p mimics significantly reduced the luciferase activity of Wt-HMGB1. However, the luciferase activity of Mut-HMGB1 was not affected by miR-505-3p mimics (Fig. 3B). Moreover, HMGB1 expression was found to be negatively correlated with miR-505-3p in glioma tissues $\left(\mathrm{P}=0.0105, \mathrm{R}^{2}=0.1825\right.$; Fig. $\left.3 \mathrm{C}\right)$. HMGB1 
A

miR-505-3p

Wild HMGB1 3'-UTR

Mutant HMGB1 3'-UTR
3'......U CCU UU GG UCGU U CACAACUG C....5'

||||||||

\section{5'...AAGUU GCAGCU GUU U U GUU GACA....3'}

5'..... AAGU U GCAGCU GUUUACAACU GA....3'
B

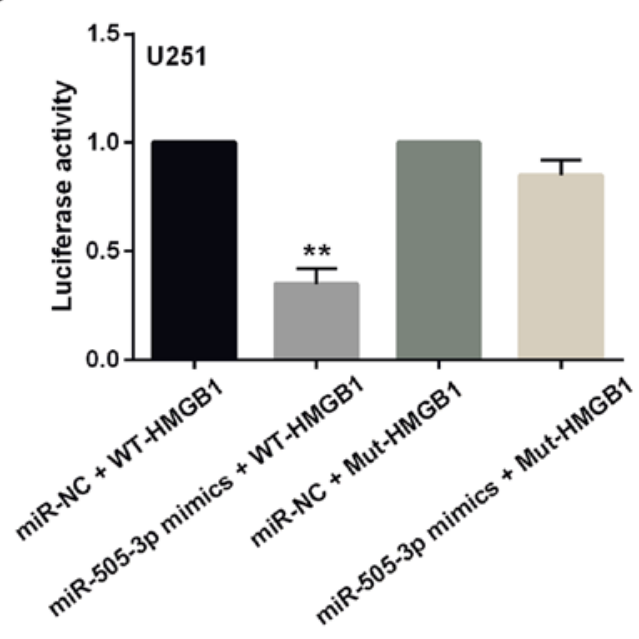

D

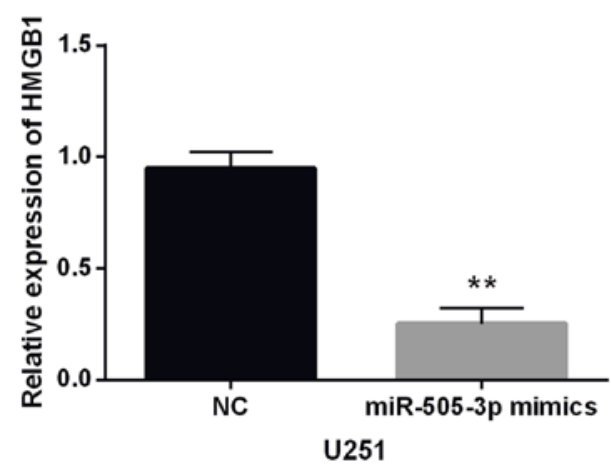

C

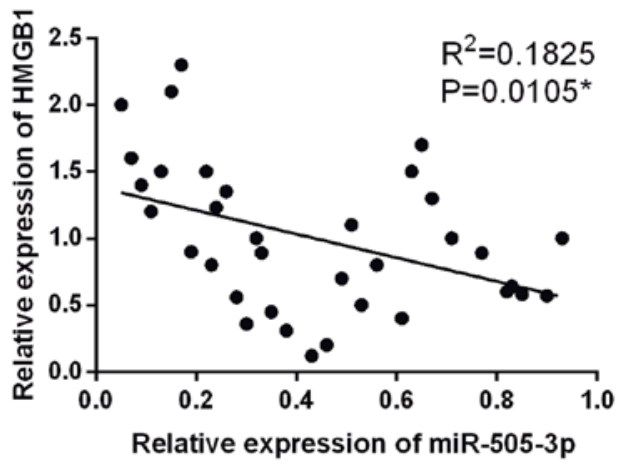

E

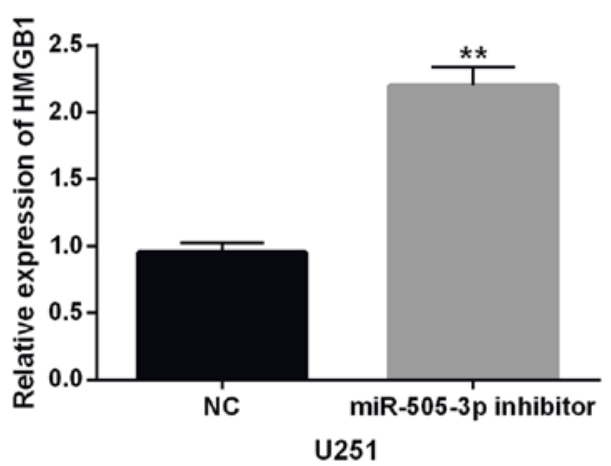

Figure 3. HMGB1 is a direct target of miR-505-3p in glioma. (A) HMGB1 has a binding site with miR-505-3p. (B) Luciferase reporter assay. (C) miR-505-3p has negative correlation with HMGB1 expression in glioma tissues. (D and E) Expression of HMGB1 was observed in U251 cells containing miR-505-3p mimics or inhibitor. ${ }^{* *} \mathrm{P}<0.01$.

expression was examined in U251 cells with miR-505-3p mimics or inhibitor. The results showed that miR-505-3p mimics reduced the expression level of HMGB1 (Fig. 3D), and miR-505-3p inhibitor enhanced the expression of HMGB1 in U251 cells (Fig. 3E). Taken together, HMGB1 is a direct target of miR-505-3p, which is inversely related to miR-505-3p expression in glioma.

HMGB1 is upregulated in glioma. Next, alteration of HMGB1 expression was identified in glioma tissues and cell lines. First, HMGB1 was found to be upregulated in the 44 cases of glioma tissues compared to adjacent normal tissues (Fig. 4A). Similarly, upregulation of HMGB1 was also identified in U251, A172 and LN229 cell lines compared to NHA cells (Fig. 4B). HMGB1 expression was associated with a prognosis in glioma patients, and high HMGB1 expression predicted a poor prognosis in glioma patients $(\mathrm{P}=0.0407$; Fig. $4 \mathrm{C})$. Therefore, we consider that HMGB1 may be involved in the development of glioma.

miR-505-3p inhibits the development of glioma through targeting $H M G B 1$. To further verify the relationship between miR-505-3p and HMGB1, HMGB1 vector was transfected into U251 cells with miR-505-3p mimics. Reduction of HMGB1 expression induced by miR-505-3p mimics was restored by HMGB1 vector in U251 cells (Fig. 5A). Then, it was found that the inhibitory effect of miR-505-3p on cell proliferation was hindered by HMGB1 vector in U251 cells (Fig. 5B). Similar results for cell migration and invasion were also identified in U251 cells (Fig. 5C and D). Collectively, miR-505-3p inhibited the development of glioma by targeting HMGB1.

miR-505-3p suppresses EMT regulating AKT expression in glioma. The effect of miR-505-3p on EMT and AKT expression 
A

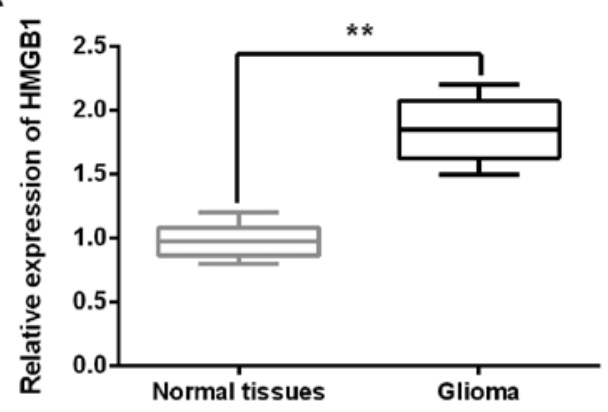

B

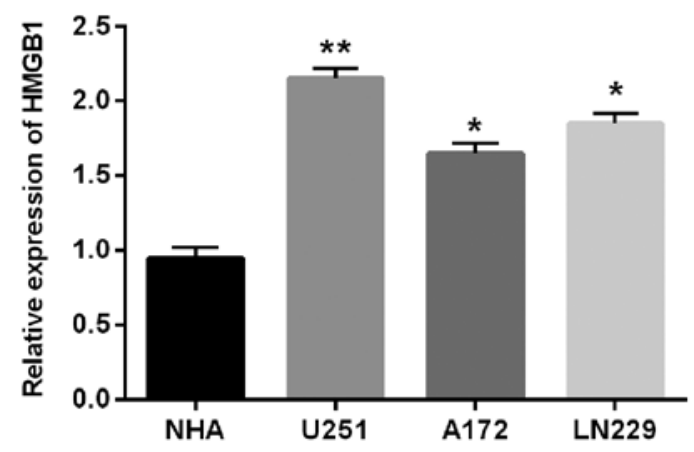

C

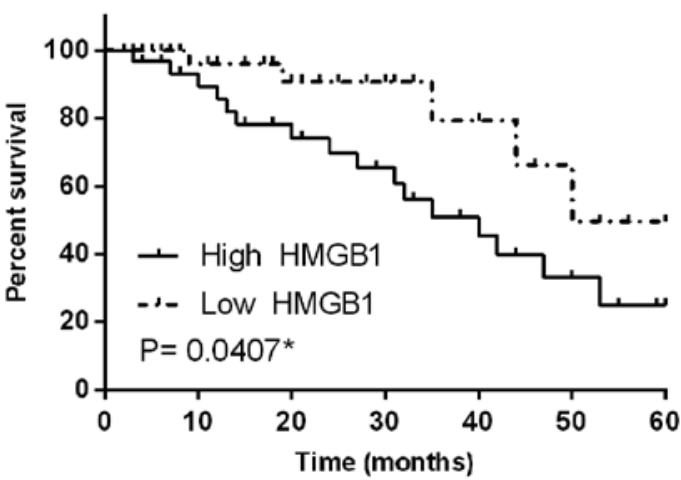

Figure 4. HMGB1 is upregulated in glioma. (A) Expression of HMGB1 in glioma tissues. (B) Expression of HMGB1 in U251, A172, LN229 and NHA cell lines. (C) High HMGB1 expression was related to shorter overall survival in glioma patients. ${ }^{*} \mathrm{P}<0.05,{ }^{* * *} \mathrm{P}<0.01$.

was investigated in glioma cells. We found that overexpression of miR-505-3p promoted E-cadherin expression and inhibited $\mathrm{N}$-cadherin and Vimentin expression in U251 cells (Fig. 6A). In contrast, knockdown of miR-505-3p had the opposite effect on these markers (Fig. 6A). Moreover, miR-505-3p mimics also promoted MMP-2 expression and suppressed MMP-9 expression. miR-505-3p inhibitor decreased MMP-2 expression and increased MMP-9 expression (Fig. 6A). Furthermore, overexpression of miR-505-3p was found to inhibit p-AKT expression (Fig. 6), while downregulation of miR-505-3p promoted p-AKT expression (Fig. 6A). However, the expression of AKT was not affected by miR-505-3p mimics or inhibitor in glioma cells. As shown in Fig. 6B, upregulation of HMGB1 weakened the effect of miR-505-3p on these makers. Briefly, miR-505-3p blocked EMT and suppressed p-AKT expression in glioma cells.

\section{Discussion}

Recently, many studies have shown that miRNAs play important roles in the pathogenesis of glioma $(19,20)$. It has been reported that miR-505-3p regulated tumorigenesis of human diseases and cancers. Especially, the combination of TMZ and miR-505 were proposed to inhibit the development of glioblastoma by modulating the WNT7B/Wnt/ $\beta$-catenin signaling pathway (21). In the current study, downregulation of miR-505-3p was detected in the 44 cases of glioma tissues (low-grade and high-grade glioma). Moreover, miR-505-3p inhibited the development of glioma by targeting HMGB1. In conclusion, miR-505-3p was identified as an inhibitory miRNA in glioma.

Consistent with our results, miR-505 was found to predict prognosis and act as tumor inhibitor in cervical carcinoma (22). Moreover, miR-505 was found to be downregulated in human osteosarcoma and suppress cell proliferation, migration and invasion (23). In this study, miR-505-3p expression was also reduced in glioma tissues, and overexpression of miR-505-3p inhibited proliferation, migration and invasion of glioma cells. Moreover, miR-505-3p also blocked EMT in glioma cells by promoting E-cadherin expression and inhibiting $\mathrm{N}$-cadherin and Vimentin expression, which has not been reported in previous studies. Furthermore, miR-505 was found to suppress proliferation and invasion of liver cancer cells by directly targeting HMGB1 (24). Our study also found that miR-505-3p inhibited the development of glioma through targeting HMGB1.

We confirmed that HMGB1 is a direct target of miR-505-3p, which is inversely related to $\mathrm{miR}-505-3 \mathrm{p}$ expression in glioma. HMGB1, as an extracellular signal molecule, has been reported to regulate tumor differentiation and metastasis (25). As an oncogene, HMGB1 was identified to promote cell proliferation and invasion in osteosarcoma (26). It was reported that knockdown of HMGB1 improved apoptosis and suppressed proliferation and invasion of glioma cells (27). Here, HMGB1 was also upregulated and functioned as an oncogene in glioma. High HMGB1 expression was identified to predict poor prognosis in glioma patients. Similarly, previous study also showed the clinical and prognostic significance of HMGB1 in human gliomas (28). Besides, upregulation of HMGB1 was found to restore the inhibitory effect of miR-505-3p in glioma.

Furthermore, we found that miR-505-3p was involved in AKT pathway by downregulating p-AKT expression in glioma. Similarly, it has been reported that miR-505 can inactivate the AKT pathway, and the AKT pathway partially was partially restored by HMGB1 in hepatocellular carcinoma (29). The AKT pathway has been proposed to be involved in the progression of many human cancers, including glioma (30). For example, tumor suppressor miRNA-34a suppressed glioma cell proliferation and tumor growth by modulating the AKT signaling pathway (31). This study showed that miR-505-3p could inactivate the AKT signaling pathway by suppressing p-AKT expression. Therefore, the inhibitory effect of miR-505-3p on proliferation, migration and invasion of glioma cells can be regulated by the HMGB1/AKT axis. However, we only explained initially the regulatory mechanism of miR-505-3p in glioma. Thus, further study is planned to address the small sample size and lack of animal experiments.

In conclusion, downregulation of miR-505-3p was identified in glioma, which was associated with shorter overall survival in glioma patients. Importantly, miR-505-3p 
A

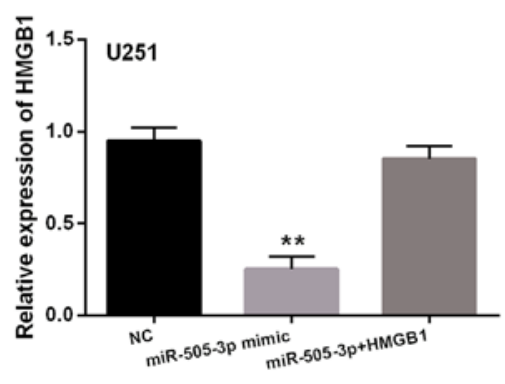

C

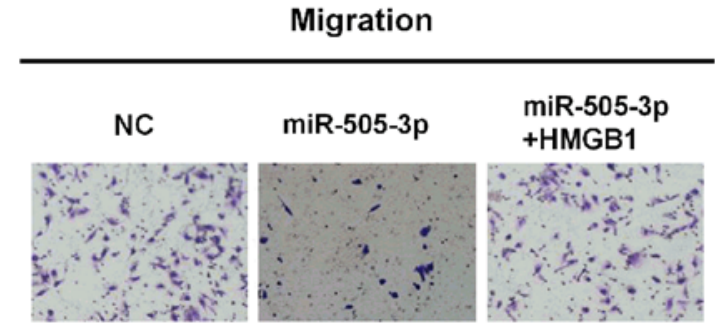

D

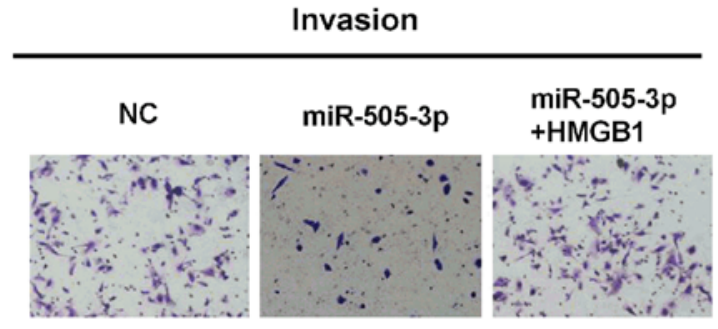

B

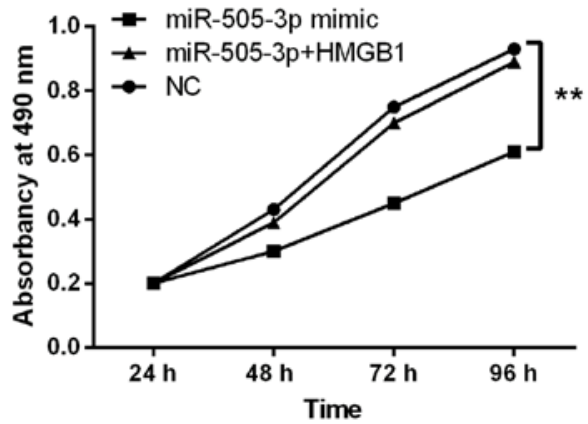

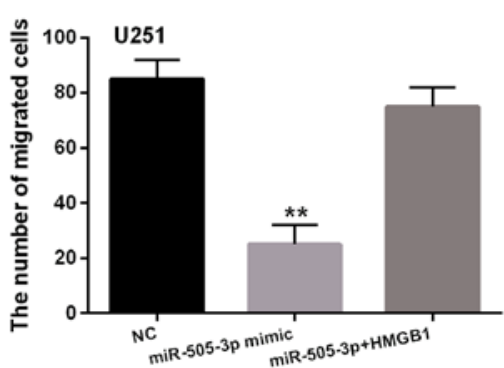

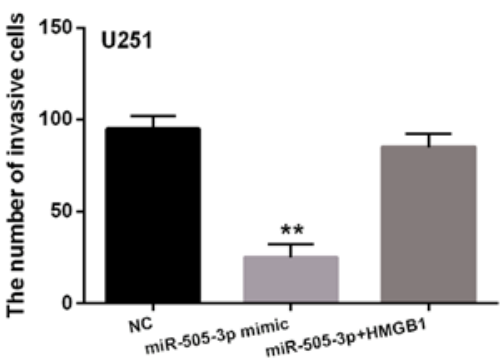

Figure 5. miR-505-3p inhibits the development of glioma through targeting HMGB1. (A) Expression of HMGB1 measured in U251 cells with HMGB1 vector and miR-505-3p mimics. (B) The cell proliferation measured in U251 cells with HMGB1 vector and miR-505-3p mimics. (C and D) Cell migration and invasion in $\mathrm{U} 251$ cells with HMGB1 vector and miR-505-3p mimics. ${ }^{* *} \mathrm{P}<0.01$

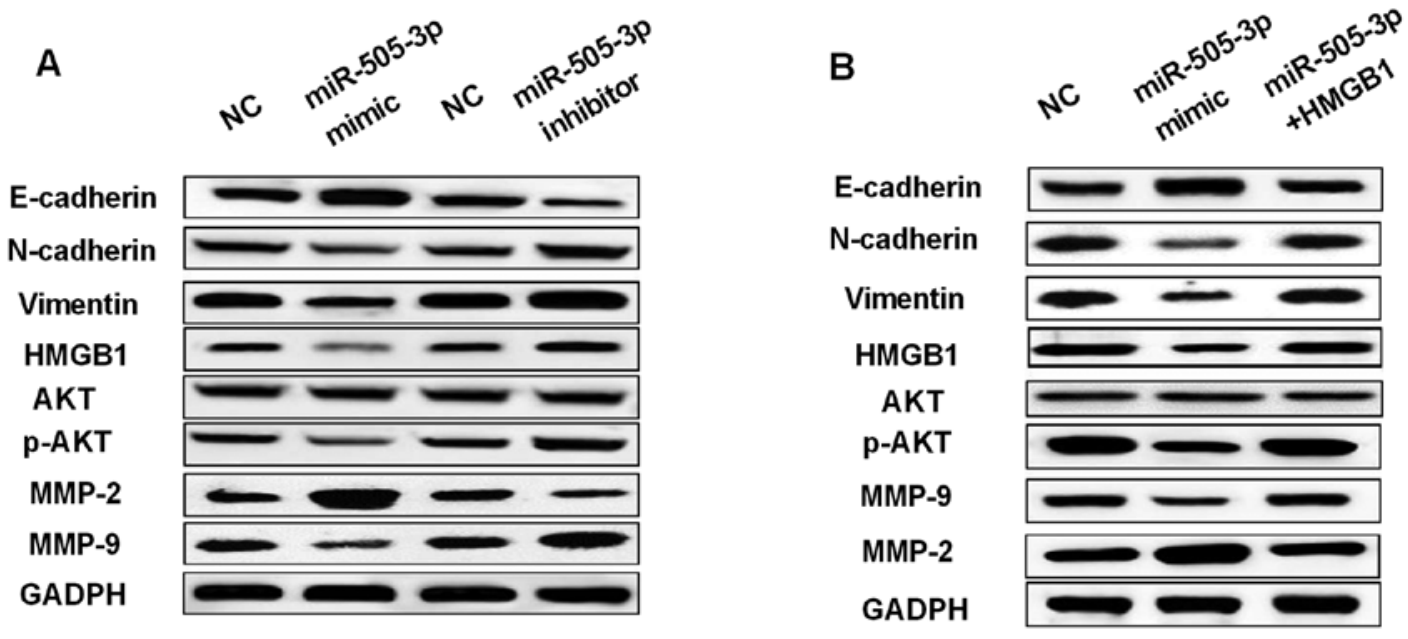

Figure 6. miR-505-3p suppresses EMT and regulating AKT expression in glioma. (A) Western blot analysis of E-cadherin, N-cadherin, Vimentin, MMP-2, MMP-9, AKT and p-AKT in U251 cells with miR-505-3p mimics or inhibitor. (B) Protein expression of E-cadherin, N-cadherin, Vimentin, MMP-2, MMP-9 and $\mathrm{p}-\mathrm{AKT}$ in U251 cells with miR-505-3p mimics and HMGB1 vector.

inhibited cell proliferation, migration and invasion in glioma by targeting HMGB1. Furthermore, miR-505-3p blocked EMT and inhibited p-AKT expression in glioma cells.

\section{Acknowledgements}

Not applicable. 


\section{Funding}

No funding was received.

\section{Availability of data and materials}

The datasets used and/or analyzed during the current study are available from the corresponding author on reasonable request.

\section{Authors' contributions}

ZC contributed to the study design, data analysis and drafted the manuscript; BW was involved in data acquisition and revision of the manuscript; $\mathrm{CZ}$ contributed to the study design, data analysis, and revised the manuscript. All authors have read and approved the final manuscript.

\section{Ethics approval and consent to participate}

The study was approved by the Institutional Ethics Committee of Department of Neurosurgery, Zhangye People's Hospital Affiliated to Hexi University (Zhangye, China). Informed consents were obtained from the patients.

\section{Patient consent for publication}

Not applicable.

\section{Competing interests}

The authors declare that they have no competing interests.

\section{References}

1. Omuro A and DeAngelis LM: Glioblastoma and other malignant gliomas: A clinical review. JAMA 310: 1842-1850, 2013.

2. Zeng T, Cui D and Gao L: Glioma: An overview of current classifications, characteristics, molecular biology and target therapies. Front Biosci 20: 1104-1115, 2015.

3. Ostrom QT, Gittleman H, Stetson L, Virk S and Barnholtz-Sloan JS: Epidemiology of intracranial gliomas. Prog Neurol Surg 30: 1-11, 2018.

4. Qaddoumi I, Sultan I and Gajjar A: Outcome and prognostic features in pediatric gliomas: A review of 6212 cases from the Surveillance, Epidemiology, and End Results database. Cancer 115: 5761-5770, 2009.

5. Bartel DP: MicroRNAs: Genomics, biogenesis, mechanism, and function. Cell 116: 281-297, 2004.

6. Wu Q, Xu L, Wang C, Fan W, Yan H and Li Q: MicroRNA-124-3p represses cell growth and cell motility by targeting EphA2 in glioma. Biochem Biophys Res Commun 503: 2436-2442, 2018.

7. Wang BQ, Yang B, Yang HC, Wang JY, Hu S, Gao YS and Bu XY MicroRNA-499a decelerates glioma cell proliferation while accelerating apoptosis through the suppression of Notch1 and the MAPK signaling pathway. Brain Res Bull 142: 96-106, 2018.

8. Xu J,He J,Huang H,Peng R and Xi J: MicroRNA-423-3p promotes glioma growth by targeting PANX2. Oncol Lett 16: 179-188, 2018.

9. Ramachandran SS, Muiwo P, Ahmad HM, Pandey RM, Singh S, Bakhshi S, Kumar L, Bhattacharya A and Gupta YK: miR-505-5p and miR-193b-3p: Potential biomarkers of imatinib response in patients with chronic myeloid leukemia. Leuk Lymphoma 58: 1981-1984, 2017.

10. Yang Q, Jia C, Wang P, Xiong M, Cui J, Li L, Wang W, Wu Q, Chen Y and Zhang T: MicroRNA-505 identified from patients with essential hypertension impairs endothelial cell migration and tube formation. Int J Cardiol 177: 925-934, 2014.

11. Qin Z, He W, Tang J, Ye Q, Dang W, Lu Y, Wang J, Li G, Yan Q and Ma J: MicroRNAs orovide feedback regulation of epithelial-mesenchymal transition induced by growth factors. J Cell Physiol 231: 120-129, 2016.
12. Ding J, Cui $X$ and Liu Q: Emerging role of HMGB1 in lung diseases: Friend or foe. J Cell Mol Med 21: 1046-1057, 2017.

13. Liu PL, Tsai JR, Hwang JJ, Chou SH, Cheng YJ, Lin FY, Chen YL, Hung CY, Chen WC, Chen YH, et al: High-mobility group box 1-mediated matrix metalloproteinase-9 expression in non-small cell lung cancer contributes to tumor cell invasiveness. Am J Respir Cell Mol Biol 43: 530-538, 2010.

14. Song B, Song WG, Li ZJ, Xu ZF, Wang XW, Wang CX and Liu J: Effect of HMGB1 silencing on cell proliferation, invasion and apoptosis of MGC-803 gastric cancer cells. Cell Biochem Funct 30: 11-17, 2012.

15. Yao X, Zhao G, Yang H, Hong X, Bie L and Liu G: Overexpression of high-mobility group box 1 correlates with tumor progression and poor prognosis in human colorectal carcinoma. J Cancer Res Clin Oncol 136: 677-684, 2010.

16. Pan C, Wang Y, Qiu MK, Wang SQ, Liu YB, Quan ZW and Ou JM: Knockdown of HMGB1 inhibits cell proliferation and induces apoptosis in hemangioma via downregulation of AKT pathway. J Biol Regul Homeost Agents 31: 41-49, 2017.

17. Zhang J, Zhang J, Qiu W, Zhang J, Li Y, Kong E, Lu A, Xu J and $\mathrm{Lu} \mathrm{X:} \mathrm{MicroRNA-1231} \mathrm{exerts} \mathrm{a} \mathrm{tumor} \mathrm{suppressor} \mathrm{role}$ through regulating the EGFR/PI3K/AKT axis in glioma. J Neurooncol 139: 547-562, 2018.

18. Livak KJ and Schmittgen TD: Analysis of relative gene expression data using real-time quantitative PCR and the 2(-Delta Delta C(T)) method. Methods 25: 402-408, 2001.

19. Ma C, Wei F, Xia H, Liu H, Dong X, Zhang Y, Luo Q, Liu Y and Li Y: MicroRNA-10b mediates TGF- $\beta 1$-regulated glioblastoma proliferation, migration and epithelial-mesenchymal transition. Int J Oncol 50: 1739-1748, 2017.

20. Jiang Y, Wang X, Zhang J and Lai R: MicroRNA-599 suppresses glioma progression by targeting RAB27B. Oncol Lett 16: 1243-1252, 2018

21. Zhang C, Yang X, Fu C and Liu X: Combination with TMZ and miR-505 inhibits the development of glioblastoma by regulating the WNT7B/Wnt/ $\beta$-catenin signaling pathway. Gene 672 : 172-179, 2018.

22. Ma C, Xu B, Husaiyin S, Wang L, Wusainahong K, Ma J, Zhu K and Niyazi M: MicroRNA-505 predicts prognosis and acts as tumor inhibitor in cervical carcinoma with inverse association with FZD4. Biomed Pharmacother 92: 586-594, 2017.

23. Liu YJ, Li W, Chang F, Liu JN, Lin JX and Chen DX: MicroRNA-505 is downregulated in human osteosarcoma and regulates cell proliferation, migration and invasion. Oncol Rep 39: 491-500, 2018.

24. Lu L, Qiu C, Li D, Bai G, Liang J and Yang Q: MicroRNA-505 suppresses proliferation and invasion in hepatoma cells by directly targeting high-mobility group box 1. Life Sci 157: 12-18, 2016.

25. Stoetzer OJ, Fersching DM, Salat C, Steinkohl O, Gabka CJ, Hamann U, Braun M, Feller AM, Heinemann V, Siegele B, et al: Circulating immunogenic cell death biomarkers HMGB1 and RAGE in breast cancer patients during neoadjuvant chemotherapy. Tumour Biol 34: 81-90, 2013.

26. Meng Q, Zhao J, Liu H, Zhou G, Zhang W, Xu X and Zheng M: HMGB1 promotes cellular proliferation and invasion, suppresses cellular apoptosis in osteosarcoma. Tumour Biol 35: 12265-12274, 2014.

27. Zhang J, Liu C and Hou R: Knockdown of HMGB1 improves apoptosis and suppresses proliferation and invasion of glioma cells. Chin J Cancer Res 26: 658-668, 2014.

28. Wang XJ, Zhou SL, Fu XD, Zhang YY, Liang B, Shou JX, Wang JY and Ma J: Clinical and prognostic significance of high-mobility group box-1 in human gliomas. Exp Ther Med 9: 513-518, 2015.

29. Lu L, Zhang D, Xu Y, Bai G, Lv Y and Liang J: miR-505 enhances doxorubicin-induced cytotoxicity in hepatocellular carcinoma through repressing the Akt pathway by directly targeting HMGB1. Biomed Pharmacother 104: 613-621, 2018.

30. Fresno Vara JA, Casado E, de Castro J, Cejas P, Belda-Iniesta C and González-Barón M: PI3K/Akt signalling pathway and cancer. Cancer Treat Rev 30: 193-204, 2004.

31. Rathod SS, Rani SB, Khan M, Muzumdar D and Shiras A: Tumor suppressive miRNA-34a suppresses cell proliferation and tumor growth of glioma stem cells by targeting Akt and Wnt signaling pathways. FEBS Open Bio 4: 485-495, 2014.

This work is licensed under a Creative Commons Attribution-NonCommercial-NoDerivatives 4.0 International (CC BY-NC-ND 4.0) License. 